\title{
Analysis of related factors of surgical treatment effect on 215 patients with laryngeal cancer
}

\author{
QIONG ZHANG ${ }^{*}$, YING XIONG ${ }^{*}$, LING LIN and KUN YUAN \\ Department of Otolaryngology, The Central Hospital of Wuhan, Tongji Medical College, \\ Huazhong University of Science and Technology, Wuhan, Hubei 430014, P.R. China
}

Received October 12, 2017; Accepted December 14, 2017

DOI: $10.3892 /$ etm.2018.5741

\begin{abstract}
This study investigated the factors affecting the surgical treatment effect on patients with laryngeal cancer. The clinical data (including the sex, age, smoking index, drinking, primary tumor site, tumor (T) stage, lymph nodes (N) stage, tumor differentiation degree, tumor diameter, surgical method, lymph node metastasis and cervical lymph node dissection) of 215 patients with laryngeal cancer in The Central Hospital of Wuhan were analyzed retrospectively; the survival rate was calculated using the Kaplan-Meier method; log-rank test was used for single-factor analysis, while Cox proportional hazard regression model was used for multiple-factor analysis. The 215 patients were followed up after surgical treatment. The 1-, 3- and 5-year survival rates were $96.2,78.0$ and $72.5 \%$, respectively. The results of singlefactor analysis showed that the age, primary tumor site, tumor differentiation degree, $\mathrm{T}$ stage, $\mathrm{N}$ stage, smoking index, tumor diameter and lymph node metastasis had significant influence on the postoperative curative effect on patients $(\mathrm{P}<0.05)$, but the patient's age, drinking and surgical method had no correlation with the postoperative curative effect $(\mathrm{P}>0.05)$. Multiple-factor analysis revealed that the primary tumor site, $\mathrm{T}$ stage, $\mathrm{N}$ stage and lymph node metastasis were the independent risk factors affecting the surgical effect on patients with laryngeal cancer. The survival rate of laryngeal cancer is decreased with the increase of $\mathrm{T}$ stage and $\mathrm{N}$ stage. The survival rate of patients with supraglottic laryngeal cancer is higher than that of patients with glottic laryngeal cancer and subglottic laryngeal cancer, and the survival rate of patients
\end{abstract}

Correspondence to: Dr Ling Lin or Dr Kun Yuan, Department of Otolaryngology, The Central Hospital of Wuhan, Tongji Medical College, Huazhong University of Science and Technology, 26 Shengli Street, Jiangan, Wuhan, Hubei 430014, P.R. China

E-mail: hbwhlinling@163.com; 421031034@qq.com

\section{${ }^{*}$ Contributed equally}

Key words: laryngeal cancer, survival rate, surgical treatment, prognosis, multiple-factor analysis with lymph node metastasis-positive laryngeal cancer is lower than that of patients with lymph node metastasis-negative laryngeal cancer. The main factors affecting the survival rate of laryngeal cancer are primary tumor site, $\mathrm{T}$ stage, $\mathrm{N}$ stage and lymph node metastasis.

\section{Introduction}

Laryngeal cancer is a kind of malignant tumor derived from the laryngeal mucosal epithelial tissues (1), and its incidence rate accounts for $5 \%$ in the incidence rate of systemic tumor and $14.2 \%$ in the incidence rate of head-neck tumors (2). In the ear-nose-throat filed, laryngeal cancer ranks third following nasopharynx cancer and nasal cancer, and its incidence rate shows an increasing trend, seriously threatening the health and life of patients $(3,4)$. Laryngeal cancer often occurs in people aged 50-70 years, more frequently in males than in females; the proportion of female patients with laryngeal cancer in the Northeast China is higher than that in official reports in China and worldwide. With the rapid development of head-neck tumor surgery, the clinical treatment techniques have been constantly developed and improved, but the prognosis is still not satisfactory (5). In order to investigate the related factors affecting the postoperative curative effect on patients with laryngeal cancer, and to develop effective therapeutic regimen and improve the survival rate of patients with laryngeal cancer, the clinical data of 215 patients with laryngeal cancer in the Central Hospital of Wuhan were retrospectively analyzed.

\section{Patients and methods}

General materials. A total of 215 patients with laryngeal cancer admitted in The Central Hospital of Wuhan from May 2010 to May 2017 were selected into the study strictly according to the inclusion and exclusion criteria. Inclusion criteria: patients without radiotherapy and chemotherapy before surgery; patients confirmed as squamous cell carcinoma via cell biopsy; patients with complete data; patients with first-onset cancer and treated in our hospital for the first time. Exclusion criteria: patients with postoperative recurrence of laryngeal cancer; patients who could not receive surgical treatment due to distant metastasis; patient who refused surgical treatment. In the 215 patients with laryngeal cancer, there were 


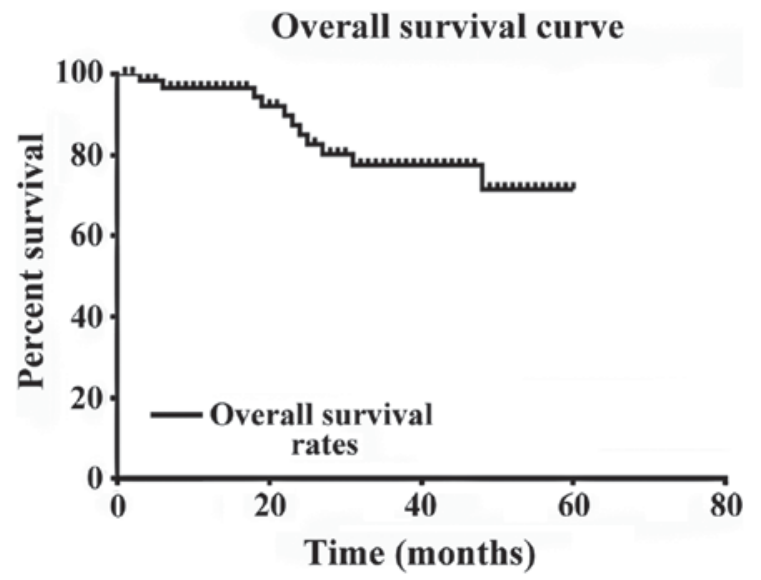

Figure 1. Postoperative survival curve of 215 patients with laryngeal cancer. It can be seen that the survival rate of patients with laryngeal cancer is decreased with time.

203 males and 12 females aged 41-80 years with an average of $57.2 \pm 3.5$ years. The vast majority of patients $(n=175)$ had a smoking history, accounting for $81.4 \%$, and approximately $62.3 \%$ patients consumed alcohol frequently. Cancer type, there were 95 cases of supraglottic laryngeal cancer, 72 cases of glottic laryngeal cancer and 48 cases of subglottic laryngeal cancer. In terms of tumor differentiation degree, there were 78 cases of high differentiation, 86 cases of moderate differentiation and 51 cases of poor differentiation. According to the latest Union for International Cancer Control (UICC) tumor lymph nodes metastasis (TNM) staging criteria, there were 48 cases of $\mathrm{T} 1$ stage, 56 cases of T2 stage, 68 cases of T3 stage and 43 cases of T4 stage. The lymph node $(\mathrm{N})$ stages were 101 cases of N0 stage, 63 cases of N1 stage and 51 cases of N2 stage. The study was approved by the Ethics Committee of The Central Hospital of Wuhan and informed consent was signed by the patients or the guardians.

Treatment methods. With surgical treatment as the basic treatment measure, all patients received clinical examinations before operation, and underwent total or partial laryngectomy through laryngoscopy and computed tomography/magnetic resonance imaging (CT/MRI) combined with the specific intraoperative situations. Of 215 patients, 148 underwent total laryngectomy, while 67 patients underwent partial laryngectomy. One hundred and fortyfour patients received cervical lymph node dissection, while 71 patients did not.

Follow-up. All patients were followed up via telephone, outpatient return visit, letters, medical data and health insurance card, with the date of operation as the starting point. The survival time began from the date of operation to the date of death, date of loss to follow-up or the last follow-up date.

Statistical analysis. SPSS 23.0 software (SPSS Inc., Chicago, IL, USA) was used for the data processing and analysis. The survival rate was calculated using Kaplan-Meier method, and the survival curve was drawn. Log-rank test was used for single-factor analysis, while Cox proportional hazard

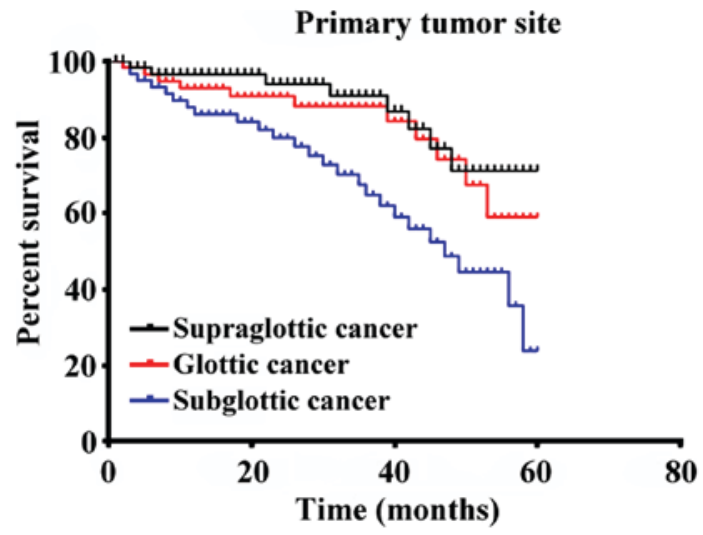

Figure 2. Postoperative survival curves of patients with different primary tumor sites. It can be seen that the survival rate of patients with supraglottic laryngeal cancer is higher than that of patients with glottic and subglottic cancers. The Kaplan-Meier method shows that the 1-, 3- and 5-year survival rates of patients with supraglottic laryngeal cancer are 95.4, 89.5 and 72.6\%, respectively. The 1-, 3- and 5-year survival rates of patients with glottic laryngeal cancer are $92.8,88.9$ and $58.3 \%$, respectively. The 1-, 3- and 5-year survival rates of patients with subglottic laryngeal cancer are 86.4, 70.8 and $22.9 \%$, respectively.

regression model was used for multiple-factor analysis. $\mathrm{P}<0.05$ was considered to indicate a statistically significant difference.

\section{Results}

Follow-up results and survival rates. All of the 215 patients were followed up, and the 5-year follow-up rate was $100 \%$. There were 16 cases of survival without tumor, 10 cases of survival with tumor and 41 cases of death, among which 21 cases died of laryngeal cancer recurrence, 2 cases died of lung metastasis, 1 case died of cervical metastasis, 4 cases died of heart disease and 13 cases died due to unknown causes. The 1-, 3- and 5-year survival rates were 96.2, 78.0 and 72.5\%, respectively, and the survival curve is shown in Fig. 1.

Single-factor analysis of postoperative curative effect on patients with laryngeal cancer. The results of single-factor analysis showed that the age, primary tumor site, tumor differentiation degree, $\mathrm{T}$ stage, $\mathrm{N}$ stage, smoking index, tumor diameter and lymph node metastasis had significant influences on the postoperative curative effect on patients $(\mathrm{P}<0.05)$, but the patient's age, drinking habit and surgical method had no significant correlation with the postoperative curative effect $(\mathrm{P}>0.05)$ (Table I).

Multiple-factor analysis of surgical effect on patients with laryngeal cancer. Cox proportional hazard regression model was used for multiple-factor analysis with the age, primary tumor site, tumor differentiation degree, $\mathrm{T}$ stage, $\mathrm{N}$ stage, smoking index, tumor diameter and lymph node metastasis in the single-factor analysis were included, and the results showed that the primary tumor site, T stage, N stage and lymph node metastasis were the independent risk factors affecting the surgical effect on patients with laryngeal cancer $(\mathrm{P}<0.05)$ (Table II). In order to show the difference in survival rate among the four independent prognostic factors, the survival curves were drawn (Figs. 2-5). 
Table I. Single-factor analysis of postoperative curative effect on 215 patients with laryngeal cancer.

\begin{tabular}{|c|c|c|c|c|c|}
\hline Characteristics & $\mathrm{n}$ & 3-year survival rate & 5-year survival rate & Log-rank value & P-value \\
\hline Sex & & & & 1.265 & 0.495 \\
\hline Male & $203(94.4 \%)$ & $81.4 \%$ & $73.1 \%$ & & \\
\hline Female & $12(5.6 \%)$ & $79.6 \%$ & $71.2 \%$ & & \\
\hline Age & & & & 7.518 & 0.038 \\
\hline$>57$ & $139(64.7 \%)$ & $65.5 \%$ & $35.2 \%$ & & \\
\hline$\leq 57$ & $76(35.3 \%)$ & $82.5 \%$ & $73.0 \%$ & & \\
\hline Smoking index (pcs/year) & & & & 7.658 & 0.029 \\
\hline$<400$ & $32(14.9 \%)$ & $85.5 \%$ & $79.4 \%$ & & \\
\hline$\geq 400,<800$ & $101(47.0 \%)$ & $72.0 \%$ & $53.5 \%$ & & \\
\hline$\geq 800$ & $82(38.1 \%)$ & $64.6 \%$ & $43.6 \%$ & & \\
\hline Primary tumor site & & & & 19.587 & $>0.05$ \\
\hline Supraglottic type & $95(44.2 \%)$ & $89.5 \%$ & $72.6 \%$ & & \\
\hline Glottic type & $72(33.5 \%)$ & $88.9 \%$ & $58.3 \%$ & & \\
\hline Subglottic type & $48(22.3 \%)$ & $70.8 \%$ & $22.9 \%$ & & \\
\hline Tumor differentiation degree & & & & 18.594 & 0.002 \\
\hline High differentiation & $78(36.3 \%)$ & $88.5 \%$ & $81.2 \%$ & & \\
\hline Moderate differentiation & $86(40.0 \%)$ & $75.6 \%$ & $67.5 \%$ & & \\
\hline Poor differentiation & $51(23.7 \%)$ & $45.1 \%$ & $43.7 \%$ & & \\
\hline T stage & & & & 17.613 & 0.013 \\
\hline $\mathrm{T} 1$ & $48(22.3 \%)$ & $91.7 \%$ & $88.4 \%$ & & \\
\hline $\mathrm{T} 2$ & $56(26.0 \%)$ & $82.1 \%$ & $79.6 \%$ & & \\
\hline $\mathrm{T} 3$ & $68(31.6 \%)$ & $61.8 \%$ & $42.1 \%$ & & \\
\hline $\mathrm{T} 4$ & $43(20.0 \%)$ & $60.5 \%$ & $40.7 \%$ & & \\
\hline $\mathrm{N}$ stage & & & & 19.142 & $>0.05$ \\
\hline N0 & $101(47.0 \%)$ & $87.1 \%$ & $76.5 \%$ & & \\
\hline N1 & $63(29.3 \%)$ & $58.7 \%$ & $49.4 \%$ & & \\
\hline N2 & $51(23.7 \%)$ & $45.1 \%$ & $31.0 \%$ & & \\
\hline Drinking & & & & 1.505 & 0.220 \\
\hline Never or occasionally & $81(37.7 \%)$ & $82.7 \%$ & $75.9 \%$ & & \\
\hline Often & $134(62.3 \%)$ & $73.1 \%$ & $65.7 \%$ & & \\
\hline Tumor diameter $(\mathrm{cm})$ & & & & 19.783 & $>0.05$ \\
\hline$\leq 2$ & $106(49.3 \%)$ & $82.1 \%$ & $76.1 \%$ & & \\
\hline$>2, \leq 4$ & $68(31.6 \%)$ & $77.9 \%$ & $49.8 \%$ & & \\
\hline$>4$ & $41(19.1 \%)$ & $70.7 \%$ & $35.7 \%$ & & \\
\hline Surgical method & & & & 6.121 & 0.059 \\
\hline Total laryngectomy & $148(68.8 \%)$ & $84.5 \%$ & $60.9 \%$ & & \\
\hline Partial laryngectomy & $67(31.2 \%)$ & $76.1 \%$ & $68.4 \%$ & & \\
\hline Lymph node metastasis & & & & 25.105 & $>0.05$ \\
\hline Positive & $46(21.4 \%)$ & $68.8 \%$ & $24.5 \%$ & & \\
\hline Negative & $169(78.6 \%)$ & $95.3 \%$ & $79.6 \%$ & & \\
\hline Cervical lymph node dissection & & & & 3.172 & 0.17 \\
\hline No & $71(33.0 \%)$ & $75.9 \%$ & $58.5 \%$ & & \\
\hline Functional & $63(29.3 \%)$ & $85.7 \%$ & $64.9 \%$ & & \\
\hline Radical & $81(37.7 \%)$ & $93.8 \%$ & $76.8 \%$ & & \\
\hline
\end{tabular}

\section{Discussion}

Laryngeal cancer is one of the common malignant tumors in the head and neck, and its incidence rate still shows an upward trend. Although progress has been made constantly in the clinical comprehensive treatment, surgery, biotherapy and genetic research, and the diagnosis and treatment of patients with laryngeal cancer are improved, its survival rate shows a 
Table II. Multiple-factor analysis of surgical effect on 215 patients with laryngeal cancer.

\begin{tabular}{lcccccc}
\hline Influencing factor & $\beta$ value & SE & Wald & P-value & Hazard ratio (HR) & 95\% confidence interval (CI) \\
\hline Primary tumor site & 1.315 & 0.368 & 10.526 & 0.001 & 3.618 & $1.592-7.925$ \\
T stage & 1.259 & 0.540 & 5.426 & 0.019 & 3.522 & $1.221-10.159$ \\
N stage & 0.699 & 0.321 & 3.200 & 0.040 & 2.012 & $1.031-3.926$ \\
Lymph node metastasis & 1.152 & 0.358 & 4.125 & 0.028 & 2.916 & $1.151-4.581$ \\
\hline
\end{tabular}

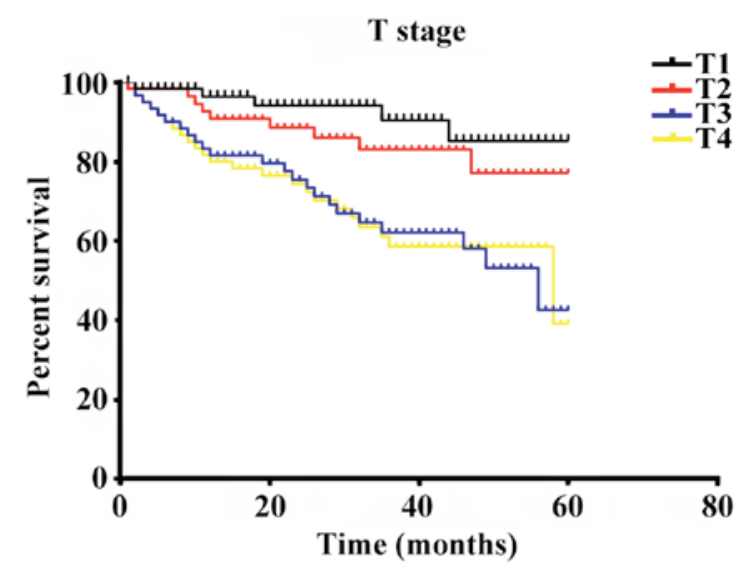

Figure 3. Survival curves of patients with different $T$ stages. The survival rates of patients in $\mathrm{T} 1$ and $\mathrm{T} 2$ stages are significantly higher than those in $\mathrm{T} 3$ and $\mathrm{T} 4$ stages.

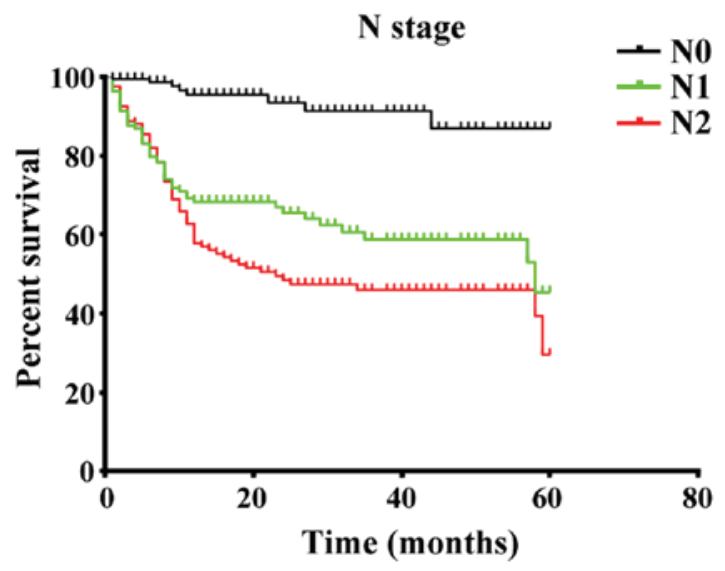

Figure 4. Survival curves of patients with different $\mathrm{N}$ stages. It can be seen that the survival rate of patients with laryngeal cancer in N0 stage is significantly higher than that in N1 and N2 stages; the 1-, 3- and 5-year survival rates of patients with laryngeal cancer in N0 stage are 95.4, 87.1 and 76.5\%, respectively. The 1-, 3- and 5-year survival rates of patients with laryngeal cancer in N1 stage are 69.5, 58.7 and $49.4 \%$, respectively. The 1-, 3- and 5 -year survival rates of patients with laryngeal cancer in N2 stage are 56.4, 45.1 and $31.0 \%$, respectively.

downward trend (6); therefore, the understanding of factors affecting the prognosis of patients, and appropriate prognosis estimation and clinical treatment are of great significance in improving the survival rate of patients with laryngeal cancer. Compared with other studies, the subjects were selected in this study in strict accordance with the inclusion and exclusion criteria for seven years, thus ensuring the credibility of results.

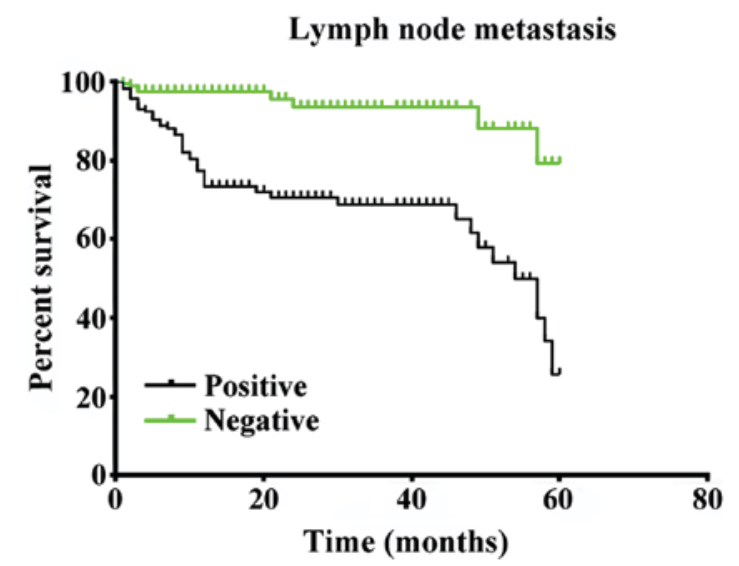

Figure 5. Survival curves of patients with lymph node metastasis. The survival rate of lymph node metastasis-negative patients is significantly higher than that of lymph node metastasis-positive patients. The 1-, 3- and 5-year survival rates of lymph node metastasis-positive patients are 73.9, 68.8 and $24.5 \%$, respectively.

The results of single-factor analysis showed that the age was an important factor affecting the patient's postoperative efficacy. This study revealed that the survival rate was $35.2 \%$ in patients with laryngeal cancer aged over 57 years and $73.0 \%$ in patients aged 57 years and below. The poorer prognosis in elderly patients than that in middle-aged patients may be caused by the following factors: the human natural resistance and the function of various organs are weakened with age; the failure of various organs lead to other types of disease and increase the mortality rate; after surgery and a series of treatment, the body function of the elderly patients is damaged, and the absorption and recovery rates are slow.

Swisher-McClure et al (7) reported that the body function declines with the age in patients with laryngeal cancer, resulting in poorer prognosis than those in the middle-aged patients than those in the middle-aged patients, and it is a potential risk factor of poor prognosis. The sex has no significant correlation with the postoperative efficacy on patients, the middle-aged men are dominant in patients with laryngeal cancer, and the incidence rate of laryngeal cancer in men is much higher than that in women, which is consistent with our research (203 male patients and 12 female patients, 17:1). The study of Guo et al (8) showed that the sex is one of the important factors affecting the prognosis of laryngeal cancer, but there was no significant correlation between sex and prognosis of laryngeal cancer in this study, and the specific influencing mechanism needs to be further explored. The smoking index is also associated with the postoperative 
efficacy on patients. The smoke in tobacco and tobacco burning contains many carcinogenic substances, and smoking has always been recognized as the most important risk factor of lung cancer (9). In the single-factor analysis, smoking also had a statistically significant effect on the postoperative efficacy on patients with laryngeal cancer.

The study of Kim et al (10) showed that the survival rate of smokers with laryngeal cancer is only one third of that of non-smokers, so smoking is an important factor affecting the prognosis of patients. In this study, both single-factor analysis and multiple-factor analysis revealed that the primary tumor site, $\mathrm{T}$ stage, $\mathrm{N}$ stage and lymph node metastasis were independent risk factors for the prognosis of patients with laryngeal cancer. Our data showed that the prognosis of patients with supraglottic cancer was superior to that of patients with glottic cancer and subglottic cancer; however, de Miguel-Luken et al (11) stated that the prognosis of patients with glottic cancer is better than that of patients with supraglottic cancer, because there are rich lymphatic vessels above the vocal cords, and lymphatic metastasis occurs easily in the tumor in this site with high occult metastasis (12). It is thought that the early symptoms of supraglottic cancer can affect the throat and pharynx, and patients will seek medical treatment once uncomfortable, which has no significant correlation with its prognosis ( $\mathrm{P}>0.05$ ); the different conclusions need further study.

It is believed that the higher the $\mathrm{T}$ stage and $\mathrm{N}$ stage are, the lower the survival rate of patients will be. The higher $\mathrm{T}$ stage indicates larger tumor size and involved range, increased lymph node metastasis rate, more damaged sites, difficult combined cure and decreased survival rate. However, Han and Ge (13) reported that the higher T stage decreases the survival rate of patients with laryngeal cancer, but it is not an independent influencing factor of prognosis. The $\mathrm{N}$ stage represents the lymph node metastasis staging of tumor, and the survival rate of patients with lymph node metastasis-positive laryngeal cancer is significantly lower than that of patients with lymph node metastasis-negative laryngeal cancer; moreover, the higher the $\mathrm{N}$ stage is, the lower the survival rate will be. The higher the tumor differentiation degree is, the malignant degree will be, reducing the survival rate of patients. Besides, the tumor diameter can reflect the tumor development time and deterioration degree indirectly, which has a significant impact on the survival rate of patients.

The study of Liu et al (14) came to the same conclusion as our study. In this study, the treatment method was not an influencing factor of prognosis, which was different from the conclusion made by Zhang et al (15), maybe because the different types of patients affect the experimental results. Zhang et al (15) thought that the correct treatment method is important for the recurrence of laryngeal cancer, the survival rate of patients receiving comprehensive treatment is higher than that of patients receiving simple surgical treatment, and the tumor can be radically cured via the combined application of radiotherapy and chemotherapy after surgical resection, greatly increasing the survival rate of patients.

In the latest study, the research on the pathogenesis and prognosis of laryngeal cancer has been developed to the levels of gene and blood type. Choe et al (16) reported that the STAT3 gene expression in laryngeal cancer cells is related to the prognosis of patients. Jin et al (17) considered that the $\mathrm{ABO}$ blood type can predict the survival rate of patients with laryngeal cancer, and the survival rate of patients with O blood type is lower than that with other types. Moreover, Dang et al (18) argued that mitochondrial DNA (mtDNA) can predict the prognosis of patients with early laryngeal cancer. These cutting-edge research directions may guide the researchers to obtain different diagnosis and quality treatment methods of laryngeal cancer.

In conclusion, we believe that the age, primary tumor site, tumor differentiation degree, $\mathrm{T}$ stage, $\mathrm{N}$ stage, smoking index, tumor diameter and lymph node metastasis are the factors affecting the surgical treatment effect on patients with laryngeal cancer. The independent factors affecting the prognosis of patients with laryngeal cancer are the primary tumor site, $\mathrm{T}$ stage, $\mathrm{N}$ stage and lymph node metastasis. Patients with laryngeal cancer should be diagnosed and treated in time, the laryngeal function should be retained as far as possible in the case of complete resection of tumor, and the surgical treatment should be combined with other treatment means, so as to improve the survival rate of patients with laryngeal cancer.

\section{Competing interests}

The authors declare that they have no competing interests.

\section{References}

1. Yu X and Li Z: The role of microRNAs expression in laryngeal cancer. Oncotarget 6: 23297-23305, 2015.

2. Du L, Li H, Zhu C, Zheng R, Zhang S and Chen W: Incidence and mortality of laryngeal cancer in China, 2011. Chin J Cancer Res 27: 52-58, 2015.

3. Li X, Wang H, Lu X and Di B: STAT3 blockade with shRNA enhances radiosensitivity in Hep-2 human laryngeal squamous carcinoma cells. Oncol Rep 23: 345-353, 2010.

4. Kim JS, Chang JW, Park JK and Hwang SG: Increased aldehyde reductase expression mediates acquired radioresistance of laryngeal cancer cells via modulating p53. Cancer Biol Ther 13: 638-646, 2012.

5. Min JW, Kim KI, Kim HA, Kim EK, Noh WC, Jeon HB, Cho DH, Oh JS, Park IC, Hwang SG, et al: INPP4B-mediated tumor resistance is associated with modulation of glucose metabolism via hexokinase 2 regulation in laryngeal cancer cells. Biochem Biophys Res Commun 440: 137-142, 2013.

6. Oancea AL, Popescu CR and Bordei P: Anatomo-surgical correlations in larynx cancer. J Med Life 5: 198-202, 2012.

7. Swisher-McClure S, Mitra N, Lin A, Ahn P, Wan F, O'Malley B, Weinstein GS and Bekelman JE: Risk of fatal cerebrovascular accidents after external beam radiation therapy for early-stage glottic laryngeal cancer. Head Neck 36: 611-616, 2014.

8. Guo W, Yang D, Xu H, Zhang Y, Huang J, Yang Z, Chen X and Huang Z: Mutations in the D-loop region and increased copy number of mitochondrial DNA in human laryngeal squamous cell carcinoma. Mol Biol Rep 40: 13-20, 2013.

9. He FY,Liu HJ, Guo Q and Sheng JL: Reduced miR-300 expression predicts poor prognosis in patients with laryngeal squamous cell carcinoma. Eur Rev Med Pharmacol Sci 21: 760-764, 2017.

10. Kim JS, Kim SY, Lee M, Kim SH, Kim SM and Kim EJ: Radioresistance in a human laryngeal squamous cell carcinoma cell line is associated with DNA methylation changes and topoisomerase II $\alpha$. Cancer Biol Ther 16: 558-566, 2015.

11. de Miguel-Luken MJ, Chaves-Conde M and Carnero A: A genetic view of laryngeal cancer heterogeneity. Cell Cycle 15: 1202-1212, 2016

12. de Miguel-Luken MJ, Chaves-Conde M, de Miguel-Luken V, Muñoz-Galván S, López-Guerra JL, Mateos JC, Pachón J, Chinchón D, Suarez V and Carnero A: MAP17 (PDZKIP1) as a novel prognostic biomarker for laryngeal cancer. Oncotarget 6: 12625-12636, 2015. 
13. Han CD and Ge WS: Up-regulation of angiotensin-converting enzyme (ace) enhances cell proliferation and predicts poor prognosis in laryngeal cancer. Med Sci Monit 22: 4132-4138, 2016.

14. Liu RR, Li MD, Li T, Tan Y, Zhang M and Chen JC: Matrix metalloproteinase 2 (MMP2) protein expression and laryngeal cancer prognosis: A meta analysis. Int J Clin Exp Med 8: 2261-2266, 2015 .

15. Zhang C, Guan Z and Peng J: The correlation between stanniocalcin 2 expression and prognosis in laryngeal squamous cell cancer. Lin Chung Er Bi Yan Hou Tou Jing Wai Ke Za Zhi 29: 102-107, 2015 (In Chinese).

16. Choe MH, Min JW, Jeon HB, Cho DH, Oh JS, Lee HG, Hwang SG, An S, Han YH and Kim JS: ERp57 modulates STAT3 activity in radioresistant laryngeal cancer cells and serves as a prognostic marker for laryngeal cancer. Oncotarget 6: 2654-2666, 2015.
17. Jin T, Li PJ, Chen XZ and Hu WH: ABO blood group is a predictor of survival in patients with laryngeal cancer. Chin $\mathrm{J}$ Cancer 35: 90, 2016.

18. Dang S, Qu Y, Wei J, Shao Y, Yang Q, Ji M, Shi B and Hou P: Low copy number of mitochondrial DNA (mtDNA) predicts worse prognosis in early-stage laryngeal cancer patients. Diagn Pathol 9: 28, 2014

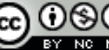

This work is licensed under a Creative Commons Attribution-NonCommercial-NoDerivatives 4.0 International (CC BY-NC-ND 4.0) License. 